\title{
B Lymphoblastic Leukemia/Lymphoma with t(v;11q23.3); KMT2A Rearranged
}

National Cancer Institute

\section{Source}

National Cancer Institute. B Lymphoblastic Leukemia/Lymphoma with t(v;11q23.3):

KMT2A Rearranged. NCI Thesaurus. Code C80332.

A precursor lymphoid neoplasm which is composed of B-lymphoblasts and carries a translocation between the KMT2A gene at 11q23.3 and another gene partner resulting in the production of a KMT2A related fusion protein. 